HEAVY VIABLE TRAJECTORIES OF A DECENTRALIZED ALLOCATION MECHANISM

Jean-Pierre Aubin

Apri1 1984

WP-84-29

Working Papers are interim reports on work of the International Institute for Applied Systems Analysis and have received only limited review. Views or opinions expressed herein do not necessarily represent those of the Institute or of its National Member Organizations.

INTERNATIONAL INSTITUTE FOR APPLIED SYSTEMS ANALYSIS A-2361 Laxenburg, Austria 
PREFACE

This paper is concerned with heavy viable trajectories of a controlled system with feedbacks and their applications to a dynamical decentralized allocation mechanism in an exchange economy.

In this framework, the controls are the prices while the states of the system are the consumption levels of the consumers. The consumption of each consumer evolves according to a differential equation controlled by the price. Viable trajectories are those which obey the ecarcity constraints: the total consumption must remain within the set of available commodities. The differential equation yielding heavy viable trajectories is constructed; providing a model of how the market may govern the evolution of prices.

This research was conducted within the framework of the Dynamics of Macrosystems study in the System and Decision Sciences Program.

ANDRZEJ WIERZBICKI

Chairman

System and Decision Sciences

Program 


\title{
HEAVY VIABLE TRAJECTORIES OF A DECENTRALIZED
}

\section{ALLOCATION MECHANISM}

\author{
Jean-Pierre Aubin \\ CEREMADE - Université de Paris-Dauphine \\ 75775 - PARIS CX (16)
}

\section{Abstract}

We define and study the concept of heavy viable trajectories of a controlled system with feedbacks in the framework a dynamical decentralized allocation mechanism in an exchange economy.

In this framework, the controls are the prices and the states of the system the consumptions of the consumers. Consumptions of each consumer evolve according a differential equation controlled by the price. Viable trajectories are the ones which obey the scarcity constraints : the sum of the consumptions must remain in the set of available commodities. Prices regulating viable trajectories evolve according a set-valued feedback map. Heavy viable trajectories are the ones associated to prices in the feedback map which evolve as slowly as possible: at each instant, the norm of the velocity of the price is minimal among the prices regulating a viable trajectory.

In this report, we construct the differential equation yielding heavy viable trajectories, providing a model of how the market may govern the evolution of prices. These results were obtained in collaboration with Halina Frankowska. 
$\underline{\text { Introduction }}$

The purpose of this report is to illustrate the concept of heavy viable trajectories of a controlled system with feedbacks - studied in Aubin-Frankowska [1984] - in the framework of an economic model of resource allocation.

Economic systems, as well as ecological and biological ones, are consuming scarce resources and face many constraints.

Therefore, the state of such systems must evolve in a viability domain defined by these sacrcity constraints as well as the other constraints. Trajectories lying in the viability domain are called viable. For instance, if we have to allocate a set of available commodities among consumers, the viability domain is the set of allocations, i.e., consumptions of consumers the sum of which are available.

Now, assume that the dynamics governing the evolution of the state are controlled. For instance, in economics, prices can be regarded as such regulating controls, giving consumers an information about the market that they use in a decentralized way to change their consumptions knowing only the state of their own consumptions. (They don't need to know neither the choices of the other consumers nor the set of available resources). Viability theory provides necessary and sufficient conditions for the existence of at least one viable trajectory starting from any viable initial state. It above all provides the feedback laws (concealed in both the dynamics and the viability domain) which relate the regulating controls -the prices- to the states of the controlled system of differential equations- the allocations of scarce resources among consumers. These feedback laws are not necessarily single-valued - deterministic -. They are most often set-valued maps, associating with each allocation a set of prices. We observe that the larger these subsets of prices are, the more flexible - and thus, the more robust - the regulation of the system will be, by allowing "mistakes" to be done. If we accept this mathematical metaphor of allocation of available commodities, we may propose that the duty of the market (Adam Smith's invisible hand) or of an adequate planning bureau should be to choose at each instant a price according to the feedback law.

In this paper, we make the further assumption that prices evolve with a high inertia : the prices will change only when the viability of the system is at stake, and then, the slower the better. This is at least the case when the market 
sets prices : the metaphorical existence of the "market" as a decision-maker should at least assume that it is lazy. And one could say the same thing about a planning bureau ! This motivates the introduction of heavy viable trajectories, associating to an allocation a price such that at each instant, the norm of the velocity is minimal among all possible prices regulating this trajectory in a viable way. We shall provide the differential equations yielding heavy trajectories (which are also concealed in the dynamics and the viability domain) and state some existence theorems.

We observe that as long as the sum of the consumptions lies in the interior of the set of available commodities, any regulatory price will work. Therefore, along a heavy trajectory, the system maintain the price inherited from the past (the regulatory prices remains constant, even though the consumptions may evolve quite rapidly).

When the sum of the consumptions reaches the boundary of the set of available resources, two situations may occur:

(a) If the sum of the velocities "points inward" the set of available commodities, then we can still keep the same regulatory price, which pushes the sum of consumptions into the set of commodities.

(b) If not, the prices will start to evolve as slowly as possible in order to pushes the total consumption back into the commodity set.

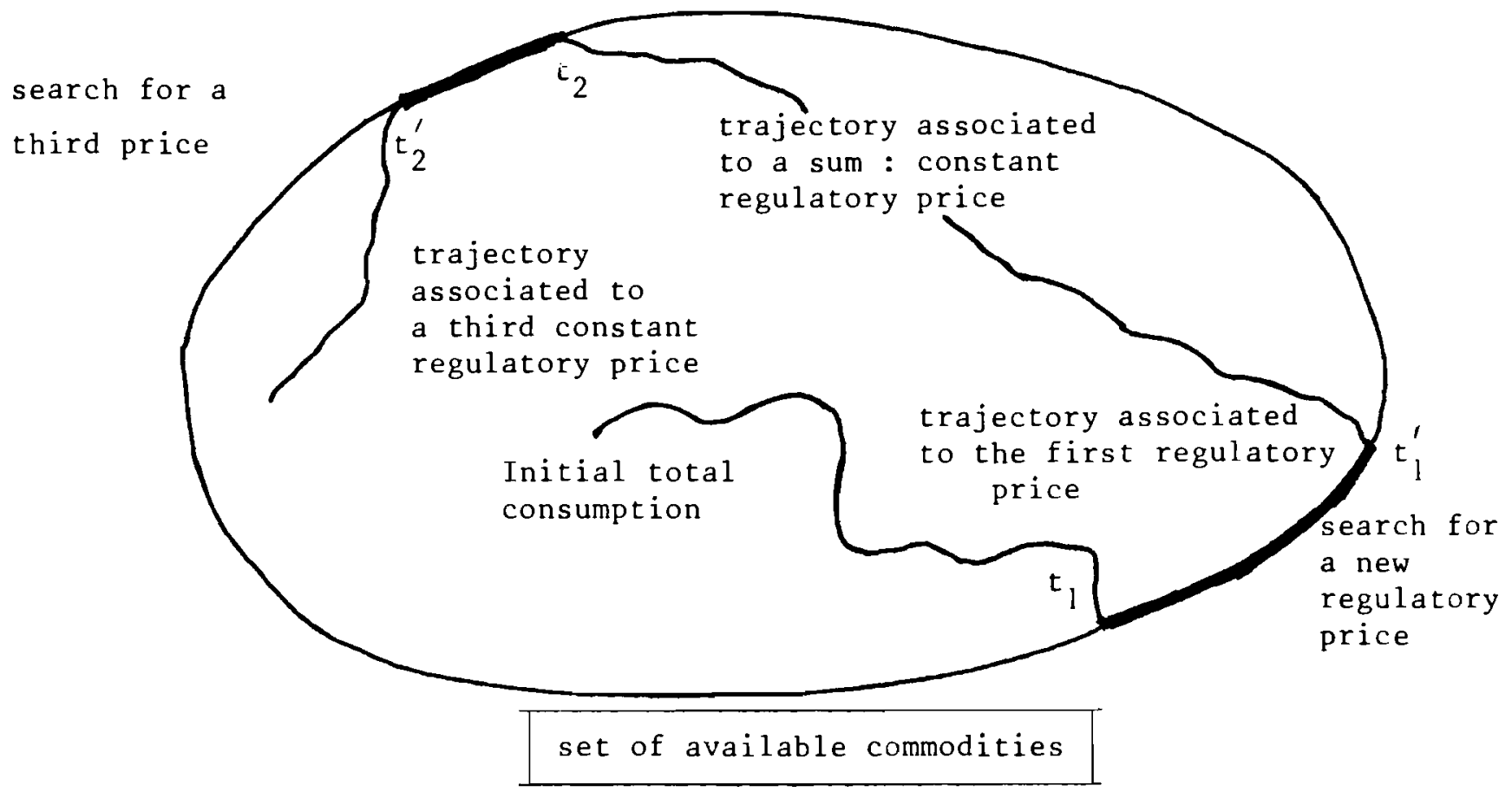




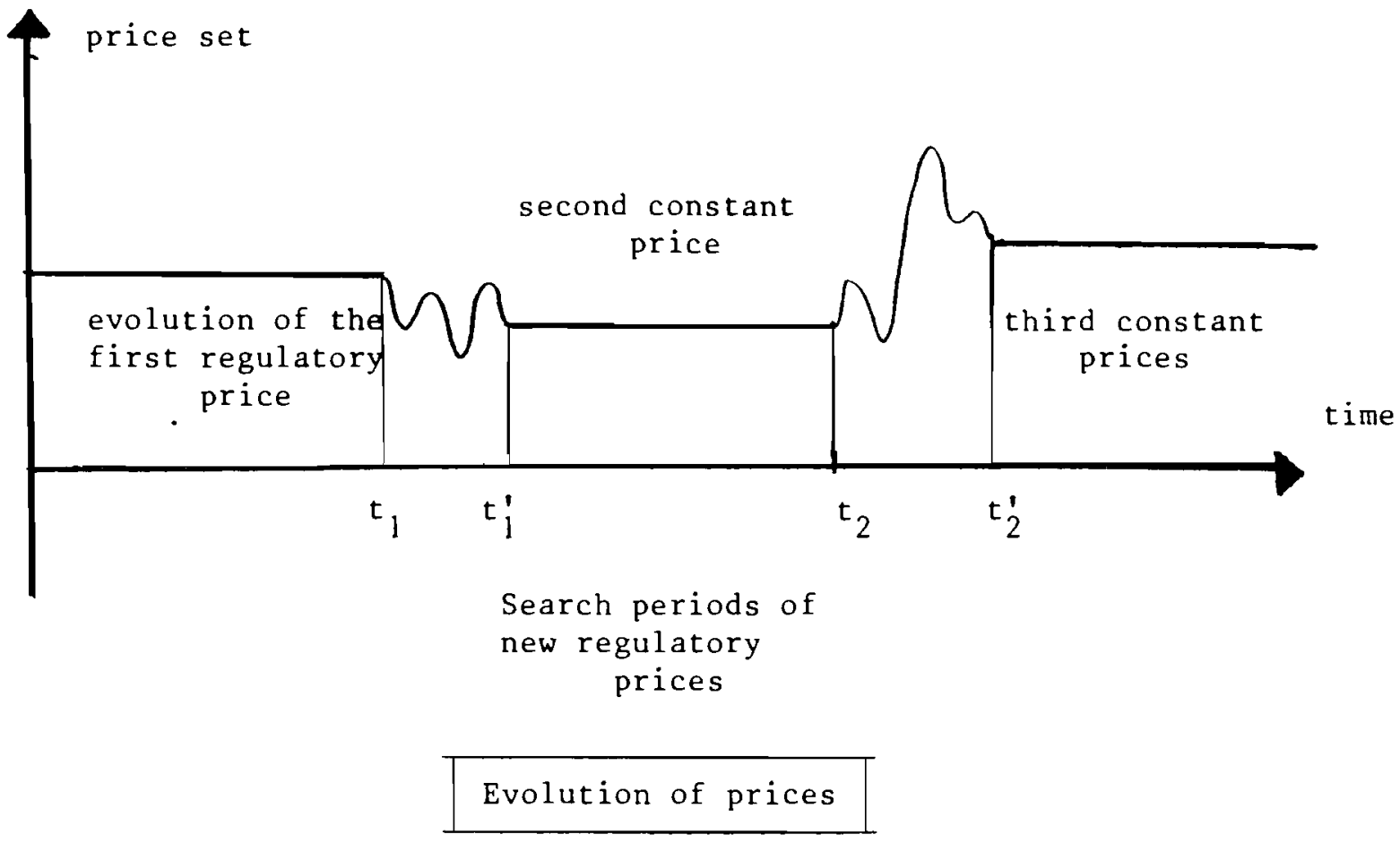

Observe that other strategies are possible, such as "enlarging" the set of available commodities instead of acting on prices. But we are far to be able to tackle all phenomena at once.

The differential equations which govern the evolution of heavy viable trajectories also reveal a division of the viability domain into "cells"; each cell is the subset of allocations which can be regulated by a constant price.

We end this informal presentation of heavy viable trajectories by mentionning that paleontological concepts of biological evolution such as punctuated equilibria proposed by Elredge and Gould are consistent with what we said.

1 . Description of the decentralized allocation mechanism.

We consider the decentralized dynamical allocation mechanism proposed in Aubin [1981 ] b) (see also Aubin-Cellina [1984] p. 245-256 and Stacchetti [1984]). We interpret $Y:=\mathbb{R}^{\ell}$ as a commodity space, its dual $Y^{\star}$ as the price space. The description of the economy begins with 
The problem is to allocate commodities $y \in M$ among $n$ consumers $i=1, \ldots, n$; each consumer chooses a commodity in its consumption set $L_{i}$.

The set $K \subset Y^{n}$ of allocations of $M$ is defined by

$$
K:=\left\{x \in \prod_{i=1}^{n} L_{i} \mid \sum_{i=1}^{n} x_{i} \in M\right\}
$$

Let $\mathrm{P} \subset \mathrm{Y}^{\star}$ denote the set of feasible prices.

We describe the behavior of each consumer by change functions $c_{i}: L_{i} X P \rightarrow Y$. The decentralized allocation mechanism is described by a system of $n$ differential equations controlled by prices : For all $x_{o}=\left(x_{o_{1}}, \ldots, x_{o_{n}}\right) \in k$, find $T>0$ and $\mathrm{n}$ absolutely continuous functions $x_{i}(\cdot)$ satisfying,

i) for almost all $t \in[0, T], \quad x_{i}^{\prime}(t)=c_{i}\left(x_{i}(t), p(t)\right)$

ii) for almost all $t \in[0, T], \quad p(t) \in P$

iii) $x_{i}(0)=x_{0}$

which are viable in the sense that

$$
\forall t \in[0, T], \forall i=1, \ldots, n, \quad x_{i}(t) \in L_{i} \quad \text { and } \quad \sum_{i=1}^{n} x_{i}(t) \in M
$$

We observe that this allocation mechanism is decentralized : the actions of each consumer depend only upon his consumption and the price.

When $K$ denotes a convex subset, we recall that the tangent cone $T_{K}(x)$ to $K$ at $x$ defined by

$$
\text { (1.5) } \quad \mathrm{T}_{\mathrm{K}}(\mathrm{x}):=\mathrm{cl}\left(\mathrm{h}_{\mathrm{h}>0}^{\mathrm{U}} \frac{1}{\mathrm{~h}}(\mathrm{~K}-\mathrm{x})\right)
$$

is a closed convex cone.

We assume that

i) the subsets $L_{i}$ and $P$ are closed and convex

ii) the subset $M$ is closed and convex and $M=M-\mathbf{R}_{+}^{\ell}$

iii) $0 \in \operatorname{Int}\left(\sum_{i=1}^{n} L_{i}-M\right)$ 
and that

$$
\left\{\begin{array}{l}
\text { i) the change functions } c_{i} \text { are } C^{1} \text { around } L_{i} \times P \\
\text { ii) } \forall x \in L_{i}, \forall p \in P, \quad c_{i}(x, p) \in T_{L_{i}}(x)
\end{array}\right.
$$

Assumptions (1.6) imply that

$$
\mathrm{T}_{\mathrm{K}}(\mathrm{x}):=\left\{\mathrm{v} \in \prod_{\mathrm{i}=1}^{\mathrm{n}} \mathrm{T}_{\mathrm{L}_{i}}\left(\mathrm{x}_{\mathrm{i}}\right) \mid \sum_{i=1}^{\mathrm{n}} \mathrm{v}_{\mathrm{i}} \in \mathrm{T}_{\mathrm{M}}\left(\sum_{i=1}^{\mathrm{n}} \mathrm{x}_{i}\right)\right\}
$$

(see Aubin-Ekeland [1984] p. 174).

We then define the feedback map $R$ from $K$ to $P$ by

$$
R(x):=\left\{p \in P \mid \sum_{i=1}^{n} c_{i}\left(x_{i}, p\right) \in T_{M}\left(\sum_{i=1}^{n} x_{i}\right)\right\}
$$

We observe that any viable trajectory of the decentralized allocation mechanism (1.3) is a solution to the feedback system : $\forall i=1, \ldots, n$,

$$
\left\{\begin{array}{lll}
\text { i) for almost all } t \in[0, T], & x_{i}^{\prime}(t)=c_{i}\left(x_{i}(t), p(t)\right) \\
\text { ii) for almost all } t \in[0, T], & p(t) \in R(x(t)) \\
\text { iii) } x_{i}(0)=x_{i_{0}} &
\end{array}\right.
$$

(see Aubin [1981] b) or Aubin-Cellina [1984 ], p. 254). The standard viability theorem (see Haddad [198] ], Aubin-Cellina [1984 ], p. 239-240) provides sufficient conditions for the existence of viable trajectories. Under the assumptions of this theorem, a necessary and sufficient condition for the existence of viable trajectories for all $x_{0} \in K$ is that

$$
\forall \mathrm{x} \in \mathrm{K} \quad, \quad \mathrm{R}(\mathrm{x}) \neq \emptyset
$$

One also shows that assumption (1.11) implies the existence of an equilibrium $\left(\bar{x}_{1}, \ldots, \bar{x}_{n}, \bar{p}\right)$, a solution to

$$
\begin{aligned}
& \text { i) } \forall i=1, \ldots, n, \quad \bar{x}_{i} \in L_{i}, \quad \sum_{i=1}^{n} \bar{x}_{i} \in M, \bar{p} \in P \\
& \text { ii) } \forall i=1, \ldots, n, \quad c_{i}\left(\bar{x}_{i}, \bar{p}\right)=0
\end{aligned}
$$


Hence, in the framework of this model, at each instant $t$, the price $p(t)$ must be chosen in the subset $R(x(t))$ : it evolves according to a set-valued feedback rule.

Then the question arises whether the market - or a planning bureau - can select a price $P(t)$ in $R(x(t))$.

We propose to answer this question by singling out heavy viable trajectory which seem to be present in the evolution of macrosystems arising in social and biological sciences (which motivated viability theory in the first place). They are trajectories which minimize at each time the norm of the velocity of the price.

The first difficulty which arises is that a solution to (1.3) is only absolutely continuous, so that the associated price is only measurable. To use the derivative in the distribution sense does not help because the concept of heavy trajectory requires the existence of the velocity of the price at almost each time. However, a straightforward strategy consists in differentiating the feedback relation $(1.10)$ ii) to reveal a law relating the velocities of the prices and the consumptions. For that purpose, we need an adequate concept of derivative of a set-valued map.

\section{2 . Contingent derivative of a set-valued map.}

When $K$ is a subset of a finite-dimensional space $X$, we can define many concepts of "tangent cones", among which we mention

a) the contingent cone $T_{K}(x)$, defined by

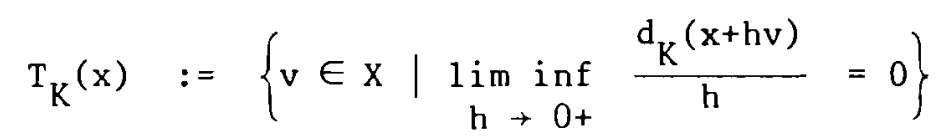

b) the tangent cone (introduced by clarke [1975])

$$
C_{K}(x):=\left\{v \in X \mid \lim _{\substack{h \rightarrow 0+\\ y \rightarrow x}} \frac{d_{K}(y+h v)}{h}=0\right\}
$$

c) the Dubovickii-Miljutin [1963] cone

$$
\left.\mathrm{D}_{\mathrm{K}}(\mathrm{x}):=\{\mathrm{v} \in \mathrm{x}|\exists \varepsilon>0| \mathrm{x}+\mid 0, \varepsilon](\mathrm{v}+\varepsilon \mathrm{B}) \subset \mathrm{K}\right\}
$$


We have the following relations (see Cornet [1981], Penot [1981 ], Aubin-Ekeland [1984] p. 409)

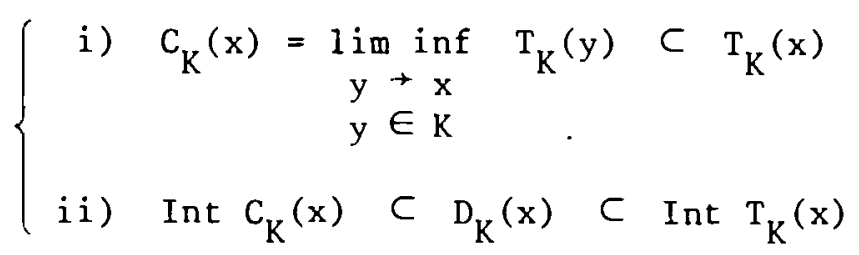

The tangent cone is always convex. It coincides with the contingent cone when $k$ is a smooth manifold (tangent space) or when $\mathrm{K}$ is convex or, more generally, when $K$ is soft in the sense that

$$
\mathrm{x} \rightarrow \mathrm{T}_{\mathrm{K}}(\mathrm{x}) \text { is lower semicontinuous. }
$$

Consider now a set-valued map $R$ from $X$ to $Y$ and a point $(x, y)$ of its graph. The contingent derivative $D R(x, y)$ is the set-valued map from $X$ to $Y$ defined by

$$
\mathrm{w} \in \operatorname{DR}(\mathrm{x}, \mathrm{y})(\mathrm{v}) \Leftrightarrow(\mathrm{v}, \mathrm{w}) \in \mathrm{T}_{\mathrm{Graph}(\mathrm{R})}(\mathrm{x}, \mathrm{y})
$$

It is equivalent to say that

$$
\lim _{\substack{h \rightarrow 0^{+} \\ v^{\prime} \rightarrow v}} d\left(w, \frac{R\left(x+h v^{\prime}\right)-y}{h}\right)=0
$$

The contingent derivative $D R(x, y)$ is a closed process (a map whose graph is a closed cone). We say that the map $R$ is soft if its graph is soft. Then $\operatorname{DR}(x, y)$ is a closed convex process, because its graph is equal to the tangent cone to Graph(R) at $(x, y)$.

We shall say that $R$ is lower semicontinuously differentiable if

$$
(x, y, v) \rightarrow D R(x, y)(v) \text { is lower semicontinuous }
$$

We observe that in this case $D R(x, y)$ is a closed convex process because property (2.8) implies that $(x, y) \rightarrow T_{G r a p h(R)}(x, y)$ is lower semicontinuous, and thus, Graph DR $(\mathrm{x}, \mathrm{y})$ is a closed convex cone.

Finally, when $K$ is a closed subset of $X$, we denote by

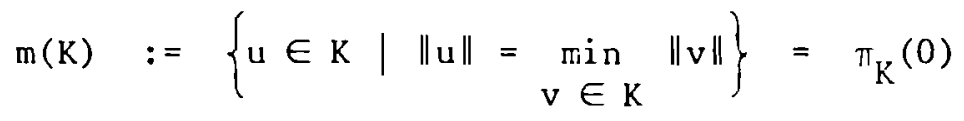


the subset of elements of $K$ with minimal norm. If $F$ is a continuous set-valued map with closed convex images, then $m(F(x))$ is reduced to a point and the single-valued map $x \rightarrow m(F(x))$ is continuous. This is no longer the case when $F$ is only upper or lower semicontinuous (with closed convex images). However,

$$
\left\{\begin{array}{l}
\text { if } F \text { is lower semicontinuous with closed images, } \\
\text { then } x \rightarrow d(0, F(x)) \text { is upper semicontinuous. }
\end{array}\right.
$$

We refer to Aubin [1983 ] and Aubin-Ekeland, [1984 ], Chapter 7, Clarke [1983 ] for a general presentation of nonsmooth analysis relevant to this study.

\section{Heavy viable trajectories.}

Let us consider the decentralized allocation mechanism (1.3), (1.4). We have seen that viable trajectories are solutions to the system (1.10). When the functions $x_{i}(\cdot)$ and $p(\cdot)$ are absolutely continuous, we deduce from the "first-order" relation (1.10)ii) the "second-order" relation

$$
\left\{\begin{array}{l}
\text { for almost all } t \in[0, T] \\
p^{\prime}(t) \in \operatorname{DR}(x(t), p(t))(c(x(t), p(t))
\end{array}\right.
$$

where we set

$$
c(x, p):=\left(c_{1}\left(x_{1}, p\right), \ldots, c_{n}\left(x_{n}, p\right)\right)
$$

Hence we can propose a rigorous definition.

\section{Definition 3.1}

We shall say that $\left(x_{1}(\cdot), \ldots, x_{n}(\cdot), p(\cdot)\right)$ is a heavy viable trajectory of the allocation mechanism $(1.3),(1.4)$ if it is a solution to the system of differential inclusions

i) $\forall i=1, \ldots, n, \quad x_{i}^{\prime}=c_{i}\left(x_{i}, p\right)$

ii) $\left.p^{\prime} \in m(D R(x, p))(c(x, p))\right)$

iii $(x(0), p(0))=\left(x_{0}, p_{0}\right)$ where $x_{0} \in k, p_{0} \in R\left(x_{0}\right)$ 
which is viable in the sense that

$$
\forall t \geqslant 0, \quad p(t) \in R(x(t))
$$

\section{Remark. Viability cells.}

The inverse of the feedback map $R$ associates with any price $p \in P$ the subset $R^{-1}(p)$ of allocations which can be regulated by $p$. The viability cells $C(p)$ are the subsets (possibly empty) of $R^{-1}(p)$ defined by

$$
C(p) \quad:=\left\{x \in R^{-1}(p) \mid 0 \in D R(x, p)(c(x, p))\right\}
$$

Starting with an allocation $\mathrm{x}_{\mathrm{o}}$ in a cell $\mathrm{c}\left(\mathrm{p}_{\mathrm{o}}\right)$ in the direction $\mathrm{c}_{\mathrm{i}}\left(\mathrm{x}_{\mathrm{o}_{\mathrm{i}}}, \mathrm{p}_{\mathrm{o}}\right)$, a heavy viable trajectory keeps the constant price $p_{o}$ as long as the allocation $x(t)$ remains in the state cell $C_{i}\left(p_{0}\right)$, because in this case the system (3.3) can be written

$$
\left\{\begin{array}{l}
\text { i) } \forall i=1, \ldots, n, \quad x_{i}^{\prime}(t)=c_{i}\left(x_{i}(t), p_{o}\right) \\
\text { ii) } 0 \in m\left(D R\left(x(t), p_{o}\right)\right)\left(c\left(x(t), p_{o}\right)\right)
\end{array}\right.
$$

The price system will start to evolve when the allocation leaves the viability cell $C\left(p_{0}\right)$.

The study of the viability problem (3.3), (3.4) runs into the same difficulties that viability problems for second-order differential inclusions encounter. Therefore, we shall use the method proposed by Cornet-Haddad [1983] to overcome these difficulties. We are ready to state our main theorem.

\section{Theorem 3.2}

We posit assumptions (1.6) and (1.7) the "transversality" condition

$$
\left\{\begin{array}{c}
\forall(y, z) \in Y x Y, \exists \pi \in T_{P}(p), \exists v_{i} \in T_{L_{i}}\left(x_{i}\right) \text { such that } \\
z=\sum_{i=1}^{n} \frac{\partial}{\partial x_{i}} c_{i}\left(x_{i}, p\right) v_{i}+\sum_{i=1}^{n} \frac{\partial}{\partial p} c_{i}\left(x_{i}, p\right) \cdot \pi \\
\quad-D T_{M}\left(\sum_{i=1}^{n} x_{i}, \sum_{i=1}^{n} c_{i}\left(x_{i}, p\right)\right)\left(\sum_{i=1}^{n} v_{i}-y\right)
\end{array}\right.
$$

and the regularity assumption 


$$
\text { the map } x \in M \rightarrow T_{M}(x) \text { is soft }
$$

Then the contingent derivative $\operatorname{DR}(x, p)(v)$ of the feedback map $R$ is the closed convex process from $\prod_{i=1}^{n} T_{L_{i}}\left(x_{i}\right)$ to $T_{P}(p)$ defined by

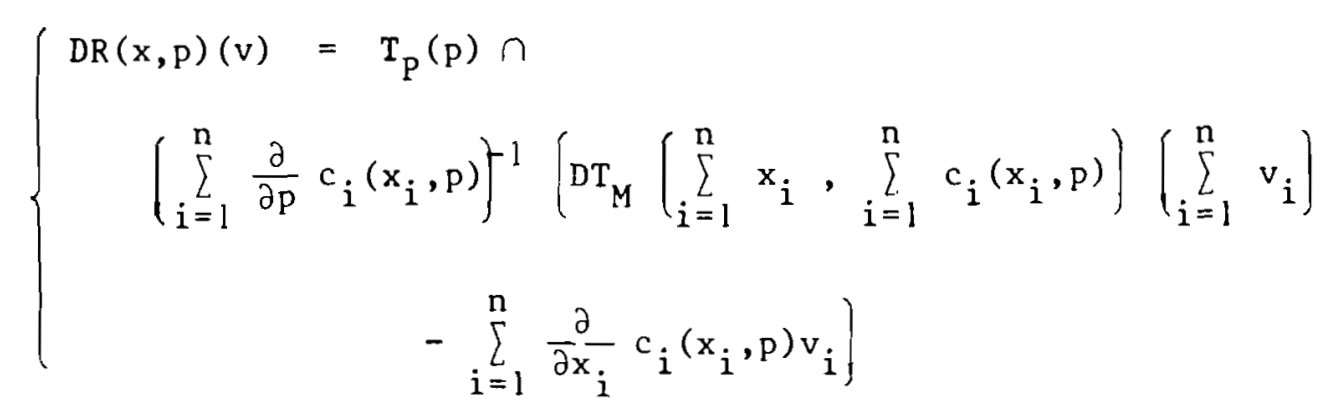

Furthermore, let us assume that

$$
\left\{\begin{array}{l}
\text { the graphs of the set-valued maps } T_{i}(\cdot) \text { and } T_{P}(\cdot) \\
\text { are locally compact }
\end{array}\right.
$$

and that

(3.11) the feedback map $R$ is lower semicontinuously differentiable If both the first order condition

$$
\forall x \in K \quad, \quad R(x) \neq \emptyset
$$

and the second order condition

$$
\forall(x, p) \in \operatorname{Graph}(R), c(x, p) \text { belongs to the domain of } D R(x, p)
$$

hold true, then for all initial allocation $x_{0} \in K$ and all initial price $P_{0}$ satisfying either

(3.14) $\quad \mathrm{p}_{\mathrm{O}} \in \mathrm{R}\left(\mathrm{x}_{\mathrm{O}}\right)$ if $\operatorname{Graph}\left(\mathrm{T}_{\mathrm{M}}(\cdot)\right)$ is locally compact

or

$$
\sum_{i=1}^{n} c_{i}\left(x_{o_{i}}, p_{o}\right) \in D_{M}\left(\sum_{i=1}^{n} x_{o_{i}}\right) \quad \text { if not }
$$

there exist $T>0$ and a heavy viable trajectory of the decentralized allocation mechanism. 


\section{Remark}

For checking assumption (3.15), we can assume that the set-valued map $T_{M}$ is lower semicontinuously differentiable and use standard theorems implying that the intersection of lower semicontinuous maps is lower semicontinuous (see AubinCellina [1984] p. 49, for instance).

\section{Example.}

We cannot have an explicit analytical expression of $m(D R(x, p)(c(x, p)))$ except in special situations. We assume, for instance, that we don't take into account the constraints on prices and consumptions of individual consumers $:$ we take $P=Y$ and $L_{i}=Y$ for all $i=1, \ldots, n$. Then, for all $x \in K$,

$$
R(x)=\left\{p \in Y^{\star} \mid \sum_{i=1}^{n} c_{i}\left(x_{i}, p\right) \in T_{M}\left(\sum_{i=1}^{n} x_{i}\right)\right\}
$$

The surjectivity property

$$
\forall(x, p) \in \operatorname{Graph}(R), \quad \sum_{i=1}^{n} \frac{\partial}{\partial p} c_{i}\left(x_{i}, p\right) \text { is surjective }
$$

implies that the transversality property (3.7) is satisfied. Hence, if we assume that $T_{M}$ is soft, we can write

$$
\left\{\begin{array}{l}
\operatorname{DR}(x, p)(v)=\left[\sum_{i=1}^{n} \frac{\partial}{\partial p} c_{i}(x, p)\right)^{-1} . \\
{\left[D T_{M}\left(\sum_{i=1}^{n} x_{i}, \sum_{i=1}^{n} c_{i}\left(x_{i}, p\right)\right)\left(\sum_{i=1}^{n} v_{i}\right)-\sum_{i=1}^{n} \frac{\partial}{\partial x_{i}} c_{i}\left(x_{i}, p\right) \cdot v_{i}\right]}
\end{array}\right.
$$

Let us set now

$$
\varphi(x, p):=\left(\sum_{i=1}^{n} \frac{\partial}{\partial p} c_{i}\left(x_{i}, p\right)\right)^{-1}\left(\sum_{i=1}^{n} \frac{\partial}{\partial x_{i}} c_{i}\left(x_{i}, p\right) c_{i}\left(x_{i}, p\right)\right)
$$

We assume that the second order condition

$$
\left\{\begin{array}{l}
\forall x \in K, \forall p \in R(x), \sum_{i=1}^{n} c_{i}\left(x_{i}, p\right) \text { belongs to the domain of } \\
\operatorname{DT}_{M}\left(\sum_{i=1}^{n} x_{i}, \sum_{i=1}^{n} c_{i}\left(x_{i}, p\right)\right)
\end{array}\right.
$$


holds true. By setting

(4.5) $\left\{\begin{array}{l}v(x, p):= \\ \left(\sum_{i=1}^{n} \frac{\partial}{\partial p} c_{i}\left(x_{i}, p\right)\right)^{-1} \operatorname{DT}_{M}\left(\sum_{i=1}^{n} x_{i}, \sum_{i=1}^{n} c_{i}\left(x_{i}, p\right)\right)\left(\sum_{i=1}^{n} c_{i}\left(x_{i}, p\right)\right)\end{array}\right.$

the element of minimal norm of $\operatorname{DR}(x, p)(c(x, p))$ can be written

$$
m(D R(x, p))(c(x, p))=\left(1-\pi_{v}(x, p)\right) \varphi(x, p)
$$

when $\pi v(x, p)$ denotes the projection of best approximation onto the closed convex subset $V(x, P)$.

The case of an exchange economy.

In order to proceed with analytical expressions, we make the further assumptions

$$
\text { (4.7) } \quad M=w-\mathbb{R}_{+}^{\ell}
$$

and

(4.8) $\quad c_{i}\left(x_{i}, p\right) \quad:=\gamma_{i}\left(x_{i}\right)-\frac{1}{n} p$

When $\gamma_{i}(x)=U_{i}^{\prime}(x)$ is the gradient of a concave differentiable utility function, then the super differential of the restriction of $U_{i}$ to a budget set $\{x \mid\langle p, x\rangle \leqslant r\}$ is $Y_{i}(x)-p \mathbb{R}_{+}$. Change functions of the form (4.8) are steepest ascent directions of utility functions restricted to budget sets.

We observe that

(4.9) $\left\{\begin{array}{l}\operatorname{DT}_{M}(y, u)(v)= \\ \left\{w \in Y \mid w_{h} \leqslant 0 \text { whenever } y_{n}=w_{h} \text { and } u_{h}=v_{h}=0\right\}\end{array}\right.$

and therefore, that $T_{M}$ is lower semicontinuously differentiable.

We set

$$
H_{l}(x):=\left\{h=1, \ldots, l \mid \sum_{i=1}^{n} x_{i h}=w_{h}\right\}
$$

and 
$(4.11)$

$$
H_{2}(x, p) \quad:=\left\{h \in H_{1}(x) \mid \sum_{i=1}^{n} c_{i}(x, p)_{h}=0\right\}
$$

They associate to each allocation $x$ and price $p$ the subsets of commodity labels for which the first and second order conditions are satisfied respectively. Indeed

and

$$
R(x):=\left\{p \in \mathbb{R} \mid p_{n} \geqslant \sum_{i=1}^{n} \gamma_{i}\left(x_{i}\right)_{h} \text { for all } h \in H_{1}(x)\right\}
$$

$$
\left\{\begin{array}{l}
D R(x, p)(c(x, p))= \\
\left\{\pi \in \mathbf{R}^{\ell} \mid \pi_{h} \geqslant \sum_{i=1}^{n}\left(\gamma_{i}^{\prime}\left(x_{i}\right)\left(\gamma_{i}\left(x_{i}\right)-\frac{1}{n} p\right)\right)_{h} \text { for all } h \in H_{2}(x, p)\right\}
\end{array}\right.
$$

Therefore

$$
\left\{\begin{array}{l}
m(D R(x, p))(c(x, p))_{h}= \\
=\left\{\begin{array}{c}
0 \text { if } h \notin H_{2}(x, p) \\
\sum_{i=1}^{n}\left(\gamma_{i}^{\prime}\left(x_{i}\right)\left(\gamma_{i}\left(x_{i}\right)-\frac{1}{n} p\right)\right)_{h} \text { if } h \in H_{2}(x, p)
\end{array}\right.
\end{array}\right.
$$

The system of differential equations governing the evolution of heavy viable allocations of $w$ are

$$
\forall i=1, \ldots, n, \quad x_{i}^{\prime}(t)=Y_{i}\left(x_{i}(t)\right)-\frac{1}{n} p(t) \quad, \quad i=1, \ldots, n
$$

and

$$
\left\{\begin{array}{l}
p_{h}^{\prime}(t)=0 \quad \text { if } h \notin H_{2}(x(t), p(t)) \\
p_{h}^{\prime}(t)=\sum_{i=1}^{n}\left(\gamma_{i}^{\prime}\left(x_{i}(t)\right)\left(\gamma_{i}\left(x_{i}(t)\right)-\frac{1}{n} p(t)\right)\right)_{h} \text { if } h \in H_{2}(x(t), p(t))
\end{array}\right.
$$

This differential equation can be interpreted as a metaphor of Adam Smith's invisible hand letting the prices evolve in this simple exchange economy. For a given commodity $h$, the price will remain constant when the total consumption $\sum_{i=1}^{n} x_{i_{h}}$ is less than the supply $w_{h}$ or when $\sum_{i=1}^{n} x_{i_{h}}=w_{h}$, but $\sum_{i=1}^{n} \gamma_{i}^{\prime}\left(x_{i}\right)_{h}<$ $<\mathrm{P}_{\mathrm{h}}$ (yielding a decrease in consumption of the commodity $h$ ). The price $\mathrm{P}_{\mathrm{n}}$ starts to evolve with minimum speed when both $\sum_{i=1}^{n} x_{i_{h}}=w_{h}$ and $\sum_{i=1}^{n} \gamma_{i}^{\prime}\left(x_{i}\right)_{h}=p_{h}$. 


\section{REFERENCES}

Aubin, J.P.

[1981 la) Contingent derivatives of set-valued maps and existence of solutions to nonlinear inclusions and differential inclusions. Advances in Mathematics. Supplementary Studies. Ed. L. Nachbin. Academic Press. 160-232.

[1981 ]b) A dynamical, pure exchange economy with feedback pricing. J. Economic Behavior and Organizations 2, 95-127.

Aubin, J.P. and A. Cellina

[1984] Differential inclusions. Springer-Verlag.

Aubin, J.P. and I. Ekeland

[1984] Applied Nonlinear Analysis. Wiley-Interscience.

Aubin, J.P. and H. Frankowska

[1984] Heavy viable trajectories of controlled systems.

Clarke, F.H.

[1975] Generalized gradients and applications. Trans. A.M.S. 205, 247-262.

[1983] Optimization and nonsmooth analysis. Wiley Interscience.

Cornet B.

[1981] Contributions à la théorie mathématique des mécanismes dynamiques d'allocation des ressources. Thèse de Doctorat d'Etat. Université de Paris-Dauphine.

Cornet, B. and G. Haddad

[1983] Théorèmes de viabilité pour les inclusions différentielles du second ordre. In Haddad's thesis, Université de Paris-Dauphine.

Dubovickii, A.I. and Miljutin, A.M.

[1963 ] Extremum problems with constraints. Soviet Math. 4, 452-455.

Ekeland, I.

[1979] Elements d'économie mathématique ; Hermann. 
Haddad, G.

[1981] Monotone trajectories of differential inclusions and functional differential inclusions with memory. Israel J. Math. 39, 83-100.

Penot, J.P.

[1981] A characterization of tangential regularity. J. Nonlinear Analysis T.M.A. 5, 625-643.

Rockafellar, R.T.

[1979] Clarke's tangent cones and the boundaries of closed sets in $R^{n}$. Nonlinear Analysis. T.M.A. 3, 145-154.

Smale, S.

[1976] Exchange processes with price adjustments. J. Math. Econ. 3, 211-216.

Stacchetti, E.

[1984] Analysis of a dynamic, decentralized exchange economy. 